\title{
Evaluation of arrhythmias in the late hospital phase of acute myocardial infarction compared to coronary care unit ectopy ${ }^{1,2}$
}

Louis A. Vismara, Anthony N. DeMaria, James L. Hughes, Dean T. Mason, and Ezra A. Amsterdam

From the Section of Cardiovascular Medicine, Departments of Medicine and Physiology, University of California, School of Medicine, Davis and Sacramento, California, U.S.A.

To evaluate the prevalence and nature of arrhythmias during the entire three-week period in hospital after myocardial infarction, the results of coronary care unit monitoring (initial 3 to 5 days) were compared with continuous 8-hour portable monitoring during the ambulatory phase (second and third weeks) in 83 consecutive survivors. Arrhythmias were detected in 84.3 per cent $(70 / 83)$ of patients while in the coronary care unit and in 85.5 per cent ( $\mathrm{I} / 83)$ during hospital stay after the coronary care unit. Ventricular ectopic depolarizations were classified as complicated (multifocal, paired, $R$ on $T$, or five or more a minute) or uncomplicated. Importantly, the high frequency of complicated ventricular extrasystoles and tachycardia persisted during the entire period in hospital (early $34.9 \%$ and late $42.2 \%$ of all patients). Howvever, only 16.9 per cent (14/83) had these ventricular arrhythmias during both coronary care unit and ward monitoring. Thus, the absence of complicated ventricular ectopic depolarization and ventricular tachycardia in the coronary care unit did not exclude their subsequent occurrence in the majority of the large number of patients with late hospital complicated ventricular ectopy.

Intensive electrocardiographic monitoring in the coronary care unit has established that arrhythmias occur in approximately 90 per cent of patients admitted to hospital with acute myocardial infarction (Imperial, Carballo, and Zimmerman, 1960; Julian, Valentine, and Miller, 1964; Spann et al., 1964; Day and Averill, 1966; Fluck et al., 1967; Mounsey, 1967; Stock, Goble, and Sloman, 1967). Continuous evaluation and aggressive management of rhythm disturbances during the initial period in hospital has significantly reduced mortality in these patients (Goble, Sloman, and Robinson, I966; Lown et al., 1967; DeSanctis, 1971). Nevertheless, sudden unexpected deaths occurring in hospital after discharge from the coronary care unit have suggested that arrhythmias may be a continuing problem (Hagstrom et al., 1967; Spracklen et al., Received 28 October 1974.

1 Presented in part at the 2rst Annual Scientific Session of The American College of Cardiology, Chicago, Ill. 3 March I972.

2 Supported in part by a Research Program Project Grant from the National Heart and Lung Institute, National Institutes of Health, Bethesda, Maryland, U.S.A.
1968; Grace and Yarvote, I97I; Paul, I97I). The development of portable electrocardiographic monitoring has provided a means of continuous recording and assessment of cardiac rhythm in ambulatory patients (Holter, 196r; Gilson, Holter, and Glasscock, 1964; Gilson, 1965; Aranaga et al., 1967). The principal purpose of this study was to evaluate arrhythmias in the late hospital period of acute myocardial infarction as compared to cardiac ectopy in the coronary care unit.

\section{Subjects and methods}

The study group consisted of 83 consecutive patients who had been admitted to hospital with acute myocardial infarction and who survived to the time of discharge three weeks after the acute event. Acute myocardial infarction was diagnosed by a typical history of chest pain, pathological $\mathbf{Q}$ waves on the electrocardiogram (except in five patients with left bundle-branch block), and characteristic increases in serum creatine phosphokinase, serum aspartate amino transferase and the cardiac isoenzyme of lactic dehydrogenase. There were 64 men and 19 women ranging in age from 39 to 84 years (average 62 years). The site of myocardial 
infarction by electrocardiographic localization of $Q$ waves was anterior in 40, inferior in 30, apical-lateral in 8 patients, and 5 patients had left bundle-branch block. All patients were admitted to the coronary care unit during the immediate period for a minimum of the initial 3 to 5 days after acute infarction, during which time continuous electrocardiographic monitoring (modified Hewlett-Packard 7800 series) was carried out in which arrhythmias were automatically detected and recorded by 24 -hour memory tape. This continuously operative system was programmed to identify changes in the $R R$ interval ( $>20 \%$ decrease) and alterations of QRS duration ( $>0.015 \mathrm{~s}$ increase) relative to immediately previous stored data, with resultant spontaneous development of rhythm strips on electrocardiographic paper during a 40-second period beginning ro seconds before and finishing 30 seconds after the electrocardiographic aberration. Further, in 7 randomly selected patients, the results of monitoring by memory loop in the coronary care unit were compared to the type of arrhythmias detected during a concurrent single period of 8-hour Holter monitoring (Avionics Electrocardiocorder, Model 350G).

Antiarrhythmic therapy was applied in the coronary care unit according to the present standards for such management. The 24 patients who manifested ventricular premature beats which were multifocal, paired, showed the $R$ on $T$ phenomenon, or were as frequent as five or more per minute, received intravenous lignocaine $100 \mathrm{mg}$ bolus followed by $\mathrm{I}$ to $4 \mathrm{mg} /$ minute maintenance for 24 hours after their disappearance. Intermittent ventricular tachycardia $(\geqslant 3$ consecutive ventricular premature beats) was treated in 5 patients by intravenous lignocaine rapid $100 \mathrm{mg}$ bolus, repeated twice at 5 -minute intervals when necessary, and sustained ventricular tachycardia (4 patients) or ventricular fibrillation (4 patients) was terminated immediately by direct current electrical cardioversion; continued suppression was then achieved by lignocaine drip I to $4 \mathrm{mg} /$ minute, with gradual transfer to oral procainamide 2 to $3 \mathrm{~g}$ daily for 3 days in the coronary care unit.

Subsequently all patients were transferred to a general medical ward. During this post-coronary care unit period of $2 \frac{1}{2}$ weeks stay in hospital, all patients underwent at least one 8-hour period of continuous portable Holter electrocardiographic monitoring. Portable monitoring was carried out during waking hours at least 9 days and no later than 18 days (average 13 days) after hospital admission. In addition, 32 randomly selected patients were monitored twice, first at I week and again at 2 weeks after leaving the coronary care unit. No patients were confined to bed during portable electrocardiographic monitoring, the degree of ambulation depending on the condition of the individual and the stage of convalescence.

In addition, at the time of post-coronary care unit monitor application the patients were interviewed and examined; arrhythmias were sought by cardiac auscultation for one full minute. A standard r2-lead electrocardiogram was obtained during the period of late hospital monitoring and the house staff physicians were questioned as to whether they had detected arrhythmias on routine clinical observations. The electromagnetic tapes obtained by portable monitoring were analysed on an electroscanner (Avionics Composite Electrocardioscanner, Model 650). All arrhythmias noted during scanning were recorded and reviewed individually by at least two of the authors.

During the 4-month intake period of this study, there were 8 deaths from cardiogenic shock in the coronary care unit. In the late hospital period, 2 patients died because of recurrent myocardial infarction with attendant pump failure, and there were 2 primary arrhythmic deaths. These 12 patients have not been included in the analysis of the present study.

\section{Results}

Arrhythmias were detected by continuous electrocardiographic memory loop in 84.3 per cent $(70 / 83$ $\mathrm{mmHg}$ ) of the patients while in the coronary care unit (Table I). Ventricular arrhythmias were most frequent $(67.5 \%$ of patients), followed by supraventricular arrhythmias $(44.6 \%)$. Second and third degree heart block were relatively uncommon (6.0\% and $2.4 \%$, respectively). Ventricular arrhythmias included ventricular fibrillation in 4 patients and ventricular tachycardia ( 3 or more consecutive ventricular beats at a rate of greater than $120 /$ minute) in 9 patients. Ventricular ectopic depolarizations were classified as complicated $(28.9 \%$ of patients) if they fulfilled any of the following criteria: I) multifocal, 2) paired, 3) $R$ on $T$ phenomenon, or 4) frequency of 5 or more per minute. Ventricular ectopic depolarizations not fulfilling these criteria were classified as uncomplicated (31.3\%). Since some patients had more than one arrhythmia, the total number of arrhythmias in Table I exceeds the number of patients.

The types of arrhythmias detected by the single period of 8-hour Holter monitoring during admission to the coronary care unit were the same as those identified by continuous memory loop monitoring in 5 of the 7 patients. Multifocal ventricular ectopic depolarizations in one patient and two episodes of paroxysmal ventricular tachycardia in another patient were detected only by the latter monitoring technique.

Post coronary care unit portable electrocardiographic monitoring for $\mathbf{8}$ hours revealed that a high frequency of arrhythmias persisted after discharge from the coronary care unit (Table 2). Arrhythmias occurred in 85.5 per cent ( $7 \mathrm{I} / 83)$ of these patients, all of whom were in the second or third week after infarction. Ventricular arrhythmias were most frequent $(66.3 \%$ of the patients) and included ventricular tachycardia $(6.0 \%)$, complicated ventricular ectopic depolarizations $(41.0 \%)$ and uncomplicated ventricular ectopic depolarizations (30.1\%). 
Twenty-eight patients ( $33.7 \%$ ) had supraventricular ectopic rhythm and one patient had second degree heart block. Only I2 patients (14.5\%) were without disturbances of cardiac rhythm after leaving the coronary care unit.

In 35 patients with complicated ventricular ectopic depolarizations shown by portable monitoring after leaving the coronary care unit, only 14 had had complicated ventricular arrhythmias or serious atrioventricular conduction disturbances while in the coronary care unit (complicated ventricular ectopic depolarizations II patients, ventricular tachycardia 5, ventricular fibrillation 3 and second degree heart block 3). In the other 21 patients only uncomplicated ventricular ectopic depolarizations (IO patients) or supraventricular arrhythmias (6 patients) were noted, and 5 patients were without arrhythmias in the coronary care unit. Of the 5 patients with ventricular tachycardia after discharge from the unit, only one had complicated ventricular ectopic depolarizations while in the unit (uncomplicated ventricular ectopic depolarizations 2 patients, atrial ectopic depolarizations $I$, and no arrhythmia I).

The results in 32 randomly selected patients monitored during the first week after leaving the coronary care unit and again 7 to Io days later are depicted in the Fig. In this group only 9.4 per cent (3/32) had complicated ventricular ectopic depolarizations during all 3 monitoring periods. Complicated ventricular ectopic depolarizations occurred in 18.8 per cent $(6 / 32)$ of patients both in the cor-

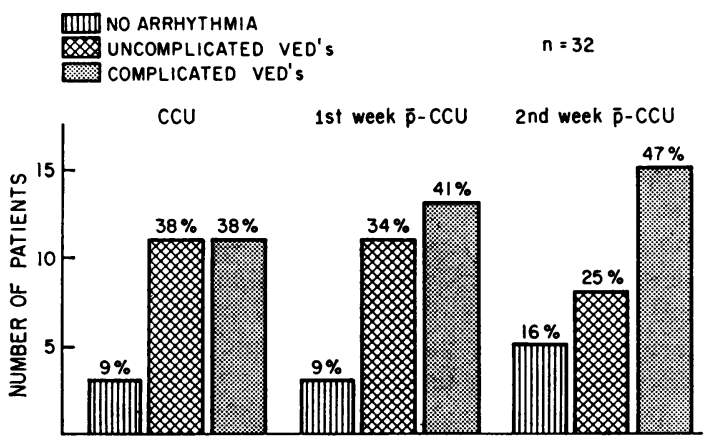

FIG. The prevalence of complicated and uncomplicated ventricular ectopic depolarizations in patients monitored sequentially while in hospital. Ambulatory monitoring (in the first and second week after leaving coronary care unit (post-CCU) was performed in the second and third week in hospital, respectively. Patients free of arrhythmias are also indicated but those with supraventricular arrhythmias are omitted. $\bar{p}-C C U=$ post-CCU; $n=$ number of patients. VED's $=$ ventricular ectopic depolarizations. onary care unit and one week later, as well as in the coronary care unit and two weeks later, while 25.0 per cent (8/32) had complicated ventricular ectopic depolarizations during both periods of ambulatory portable monitoring. Thus, while the prevalence of complicated ventricular ectopic depolarizations did not decrease over the entire period in hospital, their occurrence was variable in individual patients. The number of patients with supraventricular arrhythmias and uncomplicated ventricular ectopic depolarizations also remained essentially unchanged.

Comparison of cardiac arrhythmias detected by portable monitoring and the usual clinical methods revealed that arrhythmias documented by monitoring were usually occult and not apparent clinically. In 51 patients with monitor-detected arrhythmias, 13.7 per cent (7) described palpitations, while clinical evaluation by the primary house staff physician had detected cardiac ectopy in only 5 cases $(9.8 \%)$. Further, arrhythmias were detected in only II.8 per cent ( 6 patients) by standard electrocardiogram and in 15.7 per cent (8 patients) by cardiac auscultation for one minute. Thus, standard clinical means detected cardiac ectopy in only $12(23.3 \%)$ of the patients with monitor-detected arrhythmias.

Ventricular ectopic depolarizations were frequent even in the 31.3 per cent $(26 / 83)$ of patients who were receiving antiarrhythmic therapy during portable monitoring. Treatment in these patients consisted of either procainamide (daily dosage $\mathrm{I}$ to $3 \mathrm{~g}$ orally) or quinidine ( 0.8 to $\mathrm{r} .6 \mathrm{~g}$ orally daily). In these patients complicated ventricular ectopic depolarizations occurred in 14 subjects $(54.2 \%)$, one of whom also had episodic ventricular tachycardia. Uncomplicated ventricular ectopic depolarizations were recorded in Io patients and second degree heart block occurred once. Only one of these patients was completely free of arrhythmias during late hospital portable monitoring.

There was no correlation between arrhythmias in hospital and after leaving the coronary care unit and the patient's age, sex, location of infarction, arterial blood gases, renal function, electrolyte status, or digitalis therapy.

\section{Discussion}

In the past decade numerous reports have indicated that significant arrhythmias occur in 70 to 90 per cent of patients admitted to hospital with acute myocardial infarction (Imperial et al., I960; Julian et al., 1964; Spann et al., 1964; Day and Averill, 1966; Fluck et al., 1967; Mounsey, 1967; Stock et al., 1967; Goble et al., 1966; Lown et al., 1967; DeSanctis, 1971). These studies, however, are based on continuous electrocardiographic monitoring during the early post-infarction period in the 
coronary care unit. Though the necessity for prompt antiarrhythmic therapy in the coronary care unit is clearly established (Killip and Kimball, I967; Lown et al., I967; DeSanctis, I97I ; Killip, 1972), little is known about the incidence and significance of arrhythmias occurring during the time in hospital after leaving the coronary care unit.

The present study indicates that ventricular arrhythmias after acute myocardial infarction are not confined only to the time in the coronary care unit (Table I) but are prevalent throughout the stay in hospital (Table 2). This investigation should not be interpreted as conflicting with earlier reports which have established that lethal arrhythmias are most frequent in the initial 24 hours after acute myocardial infarction (Goble et al., 1966; Lown et al., 1967; Paul, 1971). Rather, our data extend these observations to the entire period in hospital and indicate the high incidence of ventricular electrical instability which represents a potential hazard for a significant number of patients during this period. Indeed, of all in-hospital deaths from myocardial infarction, the proportion occurring after discharge from the coronary care unit has been reported as ranging from to to 40 per cent (Goble et al., 1966; Killip and Kimball, 1967; Grace, 1970). Arrhythmias were the cause of death in approximately one-half of these patients (Grace, 1970), a conclusion that has been supported by necropsy (Adelson and Hoffman, 196r; Fulton, Julian, and Oliver, 1969; Han, 1969; Schwartz and Walsh, I97I; James, 1972).

The frequent occurrence of ventricular arrhythmias during the late hospital period of myocardial infarction is confirmed in the recent report of Moss et al. (197I). Importantly, the present study focuses attention on the value of electrocardiographic monitoring after leaving the coronary care unit since these late occurring arrhythmias were detected clinically in only 23 per cent of our patients. Physical examination, symptomatology, and twelvelead electrocardiograms were equally insensitive in identifying these arrhythmias in our experience. Thus, when ectopic beats are sought by clinical means only, their true overall frequency is considerably higher than is apparent.

The prevalence of ventricular tachycardia ( $10.8 \%$ of patients) and complicated ventricular ectopic depolarizations detected in the coronary care unit in our study is consonant with the current reported frequency of these arrhythmias, defined by the same criteria used herein, observed recently by other investigators in the early hospital phase of acute myocardial infarction: coronary care unit ventricular tachycardia Io per cent of patients (Marshall, Blount, and Genton, 1968) and 8 per cent of patients (DeSanctis, 197I). The relative reduction of ventricular tachycardia prevalence presently being observed in the coronary care unit of certain medical centres compared to its higher frequency in the past is considered to be related to the current practice of aggressive use of antiarrhythmic agents in the suppression of complicated ventricular ectopic depolarizations. Since our study was based on a comparison of early and late hospital arrhythmias treated as detailed above, the present report does not offer comment on the natural course of in-hospital disorders after acute myocardial infarction. More importantly, the present investigation evaluates the occurrence, nature, and sequence of arrhythmias throughout the hospital stay for acute myocardial infarction in the realistic setting of the application of current standards of antiarrhythmic management in this condition.

Another new finding of major importance which emerged from the present study is that patients with late occurring arrhythmias are usually not predicted by the results of coronary care unit monitoring. It is recognized that some aspects in the monitoring techniques employed varied somewhat between the early and late phases in hospital. However, our experience reported herein indicates the close correlation between the types of arrhythmias identified simultaneously by continuous monitoring and by single 8-hour period of Holter recording. Further, it is clear from the present investigation that more prolonged monitoring in the ambulatory hospital stage would not detract from our observation that serious ventricular rhythm disturbances occur in many patients $(21 / 84,25 \%)$ only during the latter part of the stay in hospital after acute myocardial infarction.

In this study complicated ventricular ectopic beats were as prevalent during ward convalescence as in the coronary care unit (Tables I and 2). The

TABLE I Arrhythmias in coronary care unit $(83$ patients)

\begin{tabular}{lll}
\hline Arrhythmia & $\begin{array}{l}\text { No. of } \\
\text { patients }\end{array}$ & $\begin{array}{c}\% \text { of } \\
\text { total }\end{array}$ \\
\hline None & 13 & 15.7 \\
Supraventricular & 37 & 44.6 \\
Ventricular & 56 & 67.5 \\
$\quad$ Ventricular tachycardia & 9 & 10.8 \\
Ventricular fibrillation & 4 & 4.8 \\
Complicated ventricular ectopic & & \\
$\quad$ depolarizations & 24 & 28.9 \\
$\quad$ Uncomplicated ectopic depolarizations & 26 & 31.3 \\
Heart block & & 6.0 \\
$\quad$ Second degree & 5 & 2.4 \\
Third degree & 2 & \\
\hline
\end{tabular}


TABLE 2 Arrhythmias after discharge from the coronary care unit ( 83 patients)

\begin{tabular}{lll}
\hline Arrhythmia & $\begin{array}{l}\text { No. of } \\
\text { patients }\end{array}$ & $\begin{array}{l}\% \text { of } \\
\text { total }\end{array}$ \\
\hline None & 12 & 14.5 \\
Supraventricular & 28 & 33.7 \\
$\begin{array}{l}\text { Ventricular } \\
\text { Ventricular tachycardia }\end{array}$ & 55 & 66.3 \\
$\quad \begin{array}{l}\text { Complicated ventricular ectopic } \\
\text { depolarizations }\end{array}$ & 5 & 6.0 \\
$\quad$ Uncomplicated ventricular ectopic & 34 & 41.0 \\
$\quad$ depolarizations & 25 & 30.1 \\
Heart block & & \\
$\quad$ Second degree & I & 1.2 \\
$\quad$ Third degree & 0 & 0 \\
\hline
\end{tabular}

prevalence of serious ventricular arrhythmias did not diminish with progressive late hospital convalescence as indicated by sequential monitoring in 32 patients, during both the second and the third hospital week after acute infarction (Fig.). The duration of these ectopics is under investigation and our preliminary data indicate that serious ventricular arrhythmias occurred in 10 of 21 asymptomatic outpatients who were monitored two months after their infarction. These data, and those of Lown and Wolf (197I) and Kotler et al. (1973), suggest that ventricular arrhythmias appear to be a chronic hazard for many patients even after successful recovery from acute myocardial infarction.

The persistence of late occurring ventricular arrhythmias may indicate that these ectopic rhythms are more closely related to myocardial ischaemia than to myocardial cell death. Indeed, in a correlative study of coronary arteriography and portable electrocardiographic monitoring, we have noted serious ventricular ectopics in over 70 per cent of patients with chronic coronary artery disease without recent infarction (Amsterdam et al., 1973). Other factors requiring further study which may be important in the genesis of late hospital arrhythmias include the role of deranged cardiac metabolism which persists throughout the healing phase of the infarct (Case, Nasser, and Crampton, 1969; Kurian and Oliver, 1970; Lovell and Prineas, 1971; Paul, I97I), as well as changes in sympathetic neurohumoral stimulation associated with either physical exertion or mental stress (Han, 1969; Jennings, 1969; Wolf, 1969).

The present study was not designed to evaluate the efficacy of antiarrhythmic therapy. However, in our study complicated ventricular ectopics were detected in 54 per cent of the patients who were receiving either quinidine or procainamide concurrently with late hospital monitoring. The limited efficacy of these oral agents in abolishing serious ventricular ectopics in our population is consistent with data recently reported in outpatients with ventricular arrhythmias (Jelinek, Lohrbauer, and Lown, 1974). The frequent adverse effects noted with therapeutic dosages of either procainamide or quinidine (Jelinek et al., 1974) further emphasize the need for future investigation to delineate clearly the indications and possible benefits of prolonged oral antiarrhythmic therapy after acute myocardial infarction.

Another critical question posed by our findings concerns the significance of the arrhythmias that persist even after a prolonged period in hospital. Though arrhythmias have been documented in asymptomatic, apparently healthy, individuals (Hinkle et al., 1967), there is increasing evidence to incriminate ventricular ectopy as an adverse prognostic factor (Chiang et al., 1969, 1970; Coronary Drug Project Research Group, 1973; Kotler et al., 1973; Moss et al., 1973). Thus, future analysis must include a prospective correlation between the data presented herein to sudden death occurring during the late hospital phase of myocardial infarction and after hospital discharge.

In conclusion, the results of our study indicate that after acute myocardial infarction serious arrhythmias are common during the entire period in hospital. The prevalence of ventricular arrhythmias with the exception of ventricular fibrillation was not significantly different in the early and late periods in hospital. The absence of significant rhythm disturbances in the coronary care unit did not exclude their presence during the latter hospital phase. Indeed, a majority of patients with late occurring ventricular ectopic depolarizations or ventricular tachycardia were free of these serious arrhythmias while in the coronary care unit. Further, serious ventricular arrhythmias persisted later in hospital in a high proportion of patients despite antiarrhythmic therapy. Since ventricular arrhythmias indicate added risk of subsequent mortality in patients with coronary artery disease, the significance, aetiology, and treatment of rhythm disturbances in the late hospital phase after myocardial infarction merit further investigation.

\section{References}

Adelson, L. A., and Hoffman, W. (I96I). Sudden death from coronary disease. Fournal of the American Medical Association, 176, 129.

Amsterdam, E. A., Vismara, L., Brocchini, R., Riggs, K., Wood, M., Massumi, R. A., Zelis, R., and Mason, D. T. (1973). Ventricular ectopic beats: relation to angiographically documented coronary artery disease. Clinical Research, 21, 399. 
Aranaga, C. E., Mower, M. M., Staewen, W. S., and Tabatznik, B. (1967). Eight-hour electrocardiogram: technique and clinical application. British Heart fournal, 29, 345.

Case, R. B., Nasser, M. G., and Crampton, R. S. (1969). Biochemical aspects of early myocardial ischemia. American fournal of Cardiology, 24, 766.

Chiang, B. N., Perlman, L. V., Fulton, M., Ostrander, L. D., and Epstein, F. H. (1970). Predisposing factors in sudden cardiac death in Tecumseh, Michigan : a prospective study. Circulation, 4I, 31 .

Chiang, B. N., Perlman, L. V., Ostrander, L. D., Jr., and Epstein, F. H. (1969). Relationship of premature systoles to coronary heart disease and sudden death in the Tecumseh epidemiologic study. Annals of Internal Medicine, 70, II 59.

Coronary Drug Project Research Group (1973). Prognostic importance of premature beats following myocardial infarction. Fournal of the American Medical Association, 223, III6.

Day, H. W., and Averill, K. (1966). Recorded arrhythmias in an acute coronary care area. Diseases of the Chest, 49, II3.

De Sanctis, R. W. (1971). Arrhythmias in myocardial infarction. Delaware Medical fournal, 43, 209.

Fluck, D. C., Olsen, E., Pentecost, B. L., Thomas, M., Fillmore, S. J., Shillingford, J. P., and Mounsey, J. P. D. (1967). Natural history and clinical significance of arrhythmias after acute cardiac infarction. British Heart fournal, 29, 170.

Fulton, M., Julian, D. G., and Oliver, M. F. (1969). Sudden death and myocardial infarction. Circulation, 39 and 40, Suppl. IV, 182.

Gilson, J. S. (1965). Electrocardiocorder-AVSEP patterns in 37 normal adult men. American fournal of Cardiology, 16, 789.

Gilson, J. S., Holter, N. J., and Glasscock, W. R. (1964). Clinical observations using the electrocardiocorderAVSEP continuous electrocardiographic system. American fournal of Cardiology, 14, 204.

Goble, A. J., Sloman, G., and Robinson, J. S. (1966). Mortality reduction in a coronary care unit. British Medical Fournal, $\mathbf{1}$, I005.

Grace, W. J. (1970). The mobile coronary care unit and the intermediate coronary care unit in the total systems approach to coronary care. Chest, 58, 363.

Grace, W. J., and Yarvote, P. M. (I97I). Acute myocardial infarction: the course of the illness following discharge from the coronary care unit. Chest, 59, 15.

Hagstrom, R. M., Billings, F. T., Jr., Ball, C. O. T., and Meneely, G. R. (1967). The risk of sudden death following myocardial infarction. Archives of Environmental Health, 15,450 .

Han, J. (1969). Mechanisms of ventricular arrhythmias associated with myocardial infarction. American fournal of Cardiology, 24, 800 .

Hinkle, L. E., Jr., Meyer, J., Stevens, M., and Carver, S. T. (1967). Tape recordings of the ECG of active men. Circulation, 36, 752.

Holter, N. J. (196I). New method for heart studies. Science, $134,1214$.

Imperial, E. S., Carballo, R., and Zimmerman, H. A. (1960). Disturbances of rate, rhythm and conduction in acute myocardial infarction. American fournal of Cardiology, $5,24$.

James, T. N. (1972). Sudden death related to myocardial infarction. Circulation, 45, 205.
Jelinek, M. V., Lohrbauer, L., and Lown, B. (1974). Antiarrhythmic drug therapy for sporadic ventricular ectopic arrhythmias. Circulation, 49, 659.

Jennings, R. B. (1969). Symposium on the pre-hospital phase of acute myocardial infarction, Part II: Early phase of myocardial ischemic injury and infarction. American Fournal of Cardiology, 24, 753.

Julian, D. G., Valentine, P. A., and Miller, G. G. (1964). Disturbances of rate, rhythm and conduction in acute myocardial infarction. American fournal of Medicine, 37, 915.

Killip, T. (1972). Management of arrhythmias in acute myocardial infarction. Hospital Practice, 7, 131 .

Killip, T., and Kimball, J. T. (1967). Treatment of myocardial infarction in a coronary care unit. American fournal of Cardiology, 20, 457.

Kotler, M. N., Tabatznik, B., Mower, M. M., and Tominaga, S. (1973). Prognostic significance of ventricular ectopic beats with respect to sudden death in the late postinfarction period. Circulation, 47, 959.

Kurian, V. A., and Oliver, M. F. (I970). Metabolic cause for arrhythmias during acute myocardial hypoxia. Lancet, $\mathbf{I}$, 813.

Lovell, R. R. H., and Prineas, R. J. (197I). Mechanisms of sudden death and their implications for prevention and management. Progress in Cardiovascular Diseases, 13, 482.

Lown, B., Fakhro, A. M., Hodd, W. B., Jr., and Thorn, G. W. (1967). The coronary care unit. Fournal of the American Medical Association, 199, 188.

Lown, B., and Wolf, M. (I97I). Approaches to sudden death from coronary heart disease. Circulation, 44, 130.

Marshall, R. M., Blount, S. G., Jr., and Genton, E. (1968). Acute myocardial infarction: influence of a coronary care unit. Archives of Internal Medicine, 122, 472.

Moss, A. J., DeCamilla, J., Hoffman, W., and Engstrom, F. (1973). Arrhythmic indicators of long term survival and late death after myocardial infarction. American fournal of Cardiology, 31, 148.

Moss, A. J., Schnitzler, R., Green, R., and DeCamilla, J. (197I). Ventricular arrhythmias 3 weeks after acute myocardial infarction. Annals of Internal Medicine, 75, 837.

Mounsey, P. (1967). Intensive coronary care; arrhythmias after acute myocardial infarction. American fournal of Cardiology, 20, 475 .

Paul, O. (I97I). Myocardial infarction and sudden death. Hospital Practice, 7, 91.

Schwartz, C. J., and Walsh, W. J. (197I). The pathologic basis of sudden death. Progress in Cardiovascular Diseases, 13, 465 .

Spann, J. F., Jr., Moellering, R. C., Jr., Haber, E., and Wheeler, E. O. (1964). Arrhythmias in acute myocardial infarction. New England fournal of Medicine, 271, 427.

Spracklen, F. H. N., Besterman, E. M. M., Everest, M. S., Litchfield, J. W., and Petrie, M. (1968). Late ventricular dysrhythmias after myocardial infarction. British Medical fournal, 4, 364 .

Stock, E., Goble, A., and Sloman, G. (1967). Assessment of arrhythmias in myocardial infarction. British Medical fournal, 2, 719.

Wolf, S. (1969). Psychosocial forces in myocardial infarction and sudden death. Circulation, 39 and 40, Suppl. IV, 74.

Requests for reprints to Dr. Louis A. Vismara, Section of Cardiovascular Medicine, University of California School of Medicine, Davis, California 956r6, U.S.A. 